$$
\begin{array}{r}
\text { Pontifícia Universidade C Católica } \\
\text { do Rio de Janeiro }
\end{array}
$$

Fábio de Azevedo Soares

\title{
Categorização Automática de Textos Baseada em Mineração de Textos
}

Tese de Doutorado

Tese apresentada como requisito parcial para obtenção do grau de Doutor pelo Programa de Pós-graduação em Engenharia Elétrica do Departamento de Engenharia Elétrica da PUC-Rio.

Orientadora: Prof. Marley M. B. R. Vellasco

Co-Orientador: Prof. Emmanuel P. L. Passos

Rio de Janeiro

Junho de 2013 


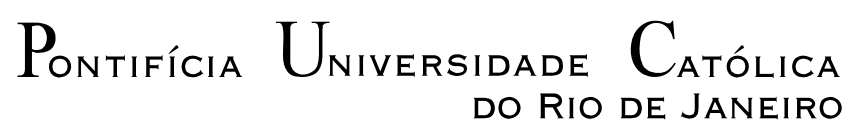

DO RIO DE JANEIRO

Fábio de Azevedo Soares

Categorização Automática de Textos Baseada em Mineração de Textos

Tese apresentada como requisito parcial para obtenção do grau Doutor pelo Programa de Pós-Graduação em Engenharia Elétrica do Departamento de Engenharia Elétrica do Centro Técnico Científico da PUC-Rio. Aprovada pela Comissão Examinadora abaixo assinada.

Profa. Marley Maria Bernardes Rebuzzi Vellasco
Orientadora
Departamento de Engenharia Elétrica - PUC-Rio

Prof. Emmanuel Piseces Lopes Passos

Co-Orientador

Aposentado do IME

Profa. Karla Tereza Figueiredo Leite

UEZO

Prof. Rubens Nascimento Melo

Departamento de Informática -PUC-Rio

Prof. Ronaldo Ribeiro Goldschmidt

UFRRJ

Prof. Douglas Mota Dias

Departamento de Engenharia Elétrica - PUC-Rio

Prof. Cláudio Márcio do Nascimento Abreu Pereira

Comissão Nacional de Energia Nuclear

Prof. José Eugenio Leal

Coordenador Setorial do Centro

Técnico Científico - PUC-Rio

Rio de Janeiro, 10 de Junho de 2013 
Todos os direitos reservados. É proibida a reprodução total ou parcial do trabalho sem autorização da universidade, do autor e do orientador.

Fábio de Azevedo Soares

Graduou-se Bacharel em Ciência da Computação em 2006. Mestre pela PUC-Rio em 2008. Atua como Desenvolvedor de Softwares, principalmente no desenvolvimento de Sistemas de Apoio à Decisão. Leciona para o ensino universitário. Tem interesse na pesquisa de novos algoritmos, principalmente, na área de Mineração de Textos e Aprendizado de Máquina.

\section{Ficha Catalográfica}

Soares, Fábio de Azevedo

Categorização automática de textos baseada em mineração de textos / Fábio de Azevedo Soares ; orientadores: Marley M. B. R. Vellasco, Emmanuel P. L. Passos. - 2013. $158 \mathrm{f.} ; 30 \mathrm{~cm}$ Tese (doutorado)-Pontifícia Universidade Católica do Rio de Janeiro, Departamento de Engenharia Elétrica, 2013. Inclui bibliografia

1. Engenharia elétrica - Teses. 2. Mineração de textos. 3. Categorização. 4. Framework. 5. Português brasileiro. 6. Automática. I. Vellasco, Marley M. B. R. II. Passos, Emmanuel P. L. III. Pontifícia Universidade Católica do Rio de Janeiro. Departamento de Engenharia Elétrica. IV. Título. 


\section{Agradecimentos}

A Deus por ser e pela oportunidade de todo dia começar de novo.

A Gabriela Soares por dar um novo sentido a minha vida, ser a minha fonte de amor e esperança, ensinar mais do que posso aprender e pelo sorriso de cada dia.

Ao professor Emmanuel Passos pelo apoio, dedicação, paciência, amizade e inspiração de vida.

À professora Marley Vellasco pela confiança depositada, oportunidade de realizar este trabalho e exemplo de mestre.

Aos meus pais pelo carinho, preocupação e expectativa de conclusão deste trabalho.

Ao meu amigo Thiago Mendonça por incentivar e compreender mais do que precisava.

Ao CNPq e à CAPES pelo apoio financeiro.

À PUC-Rio e à Vice Reitoria Acadêmica (VRAc) pela bolsa de isenção que me foi concedida. 


\section{Resumo}

Soares, Fábio de Azevedo; Vellasco, Marley Maria Bernardes Rebuzzi (Orientadora); Passos, Emmanuel Piseces Lopes Passos (Co-Orientador).

Categorização Automática de Textos Baseada em Mineração de Textos. Rio de Janeiro, 2013. 158p. Tese de Doutorado - Departamento de Engenharia Elétrica, Pontifícia Universidade Católica do Rio de Janeiro.

A Categorização de Documentos, uma das tarefas desempenhadas em Mineração de Textos, pode ser descrita como a obtenção de uma função que seja capaz de atribuir a um documento uma categoria a que ele pertença. O principal objetivo de se construir uma taxonomia de documentos é tornar mais fácil a obtenção de informação relevante. Porém, a implementação e a execução de um processo de Categorização de Documentos não é uma tarefa trivial: as ferramentas de Mineração de Textos estão em processo de amadurecimento e ainda, demandam elevado conhecimento técnico para a sua utilização. Além disso, exercendo grande importância em um processo de Mineração de Textos, a linguagem em que os documentos se encontram escritas deve ser tratada com as particularidades do idioma. Contudo há grande carência de ferramentas que forneçam tratamento adequado ao Português do Brasil. Dessa forma, os objetivos principais deste trabalho são pesquisar, propor, implementar e avaliar um framework de Mineração de Textos para a Categorização Automática de Documentos, capaz de auxiliar a execução do processo de descoberta de conhecimento e que ofereça processamento linguístico para o Português do Brasil.

\section{Palavras-chave}

Mineração de Textos; Categorização; Framework; Português brasileiro; Automática. 


\section{Abstract}

Soares, Fábio de Azevedo; Vellasco, Marley Maria Bernardes Rebuzzi (Advisor); Passos, Emmanuel Piseces Lopes Passos (Co-Advisor). Automatic Text Categorization Based on Text Mining. Rio de Janeiro, 2013. 158p. Ph.D. Thesis - Departamento de Engenharia Elétrica, Pontifícia Universidade Católica do Rio de Janeiro.

Text Categorization, one of the tasks performed in Text Mining, can be described as the achievement of a function that is able to assign a document to the category, previously defined, to which it belongs. The main goal of building a taxonomy of documents is to make easier obtaining relevant information. However, the implementation and execution of Text Categorization is not a trivial task: Text Mining tools are under development and still require high technical expertise to be handled, also having great significance in a Text Mining process, the language of the documents should be treated with the peculiarities of each idiom. Yet there is great need for tools that provide proper handling to Portuguese of Brazil. Thus, the main aims of this work are to research, propose, implement and evaluate a Text Mining Framework for Automatic Text Categorization, capable of assisting the execution of knowledge discovery process and provides language processing for Brazilian Portuguese.

\section{Keywords}

Text Mining; Text Categorization; Framework; Brazilian Portuguese; Automatic. 


\section{Sumário}

1 Introdução 16

1.1. Motivação 16

1.2. Objetivos do Trabalho 20

1.3. Trabalhos relacionados 21

1.4. Organização da Tese 28

2 Mineração de Textos: Fundamentos 30

2.1. Definição 30

2.2. Principais Elementos 34

2.3. Documentos textuais são estruturados 36

2.4. Características representativas de um documento 37

2.5. Abordagens ao processo de Mineração de Textos 40

2.5.1. Análise Estatística $\quad 40$

2.5.2. Análise Semântica $\quad 41$

2.6. Áreas correlatas a Mineração de Textos 42

2.6.1. Ciência Cognitiva 42

2.6.2. Processamento de Linguagem Natural 43

2.6.3. Aprendizado de Máquina $\quad 44$

2.6.4. Estatística $\quad 45$

2.6.5. Recuperação de Informação 46

2.6.6. Mineração de Dados 47

3 Metodologia de Mineração de Textos 49

3.1. Coleta de Dados $\quad 50$

3.2. Pré-Processamento 51

3.2.1. Tokenização

3.2.2. Remoção de stopwords

3.2.3. Processamento de Linguagem Natural 55

3.3. Indexação $\quad 62$

3.3.1. Indexação Textual

3.3.2. Indexação Temática $\quad 64$

3.4. Mineração 65

3.5. Análise 66

$\begin{array}{ll}\text { 3.5.1. Precisão } & 68\end{array}$ 
3.5.2. Abrangência 68

3.5.3. Medida-F $\quad 69$

3.5.4. Precisão x Abrangência 69

4 Recuperação de Informação

4.1. Introdução

4.2. Histórico da área de Recuperação de Informação 73

4.2.1. $1^{\text {a }}$ Fase - Décadas de 50 e 60

4.2.2. $2^{\mathrm{a}}$ Fase - Décadas de 70 e 80

4.2.3. $3^{\text {a }}$ Fase - Década de 90 em diante $\quad 74$

4.3. Recuperação de Informação Clássica 75

4.3.1. Modelos de Representação de Documentos 77

5 Categorização de Textos $\quad 86$

5.1. Introdução 86

5.2. Histórico da área de Categorização de Textos 87

5.2.1. $1^{\text {a }}$ Fase - Até o final década de 80

5.2.2. $2^{\mathrm{a}}$ Fase - Década de 90 em diante 87

5.3. Definição 88

5.4. Tipos de Classificadores $\quad 89$

5.5. Modelagem da categorização 90

5.6. Tipos de categorização 91

5.7. Aplicações de Categorização de Textos 92

5.7.1. Organização de documentos 92

5.7.2. Filtragem de Documentos $\quad 92$

5.7.3. Desambiguação Lexical de Sentido 93

5.8. Aprendizagem de Máquina em CT 94

5.8.1. Aprendizagem Supervisionada $\quad 94$

5.8.2. Treinamento e Teste 95

5.8.3. $k$-Nearest Neighbors 96

5.8.4. SVM 97

5.8.5. Combinação de Classificadores 100

5.9. Ferramentas de Mineração de Textos 103

5.9.1. Weka 103

5.9.2. Text Mine 104

$\begin{array}{lr}\text { 5.9.3. TMSK } & 105\end{array}$

5.9.4. RIKTEXT 106

5.9.5. STATISCA Text Miner 106

$\begin{array}{ll}6 \text { Framework proposto } & 109\end{array}$

6.1. Definição 109 
$\begin{array}{ll}\text { 6.2. Ambiente de desenvolvimento } & 110\end{array}$

$\begin{array}{ll}\text { 6.3. Objetivos } & 110\end{array}$

$\begin{array}{ll}\text { 6.4. Coleta } & 111\end{array}$

6.5. Pré-Processamento 111

6.5.1. Tokenização 111

6.5.2. Análise/Remoção de stopwords 112

6.5.3. Processamento de Linguagem Natural 114

6.5.4. Redução de características 121

6.5.5. Indexação 122

6.5.6. Classificadores implementados 122

6.5.7. Técnicas de combinação de classificadores 123

6.6. Corpus 123

6.7. Assistência Inteligente 126

7 Estudos de Caso 129

7.1. Coleta 129

7.2. Treinamento 129

$\begin{array}{ll}\text { 7.3. Resultados } & 129\end{array}$

7.3.1. Tokenização 129

7.3.1. Remoção de stopwords 132

7.3.2. PLN - Identificação de classes gramaticais 134

7.3.3. PLN - Lematização 135

$\begin{array}{ll}\text { 7.3.4. Thesaurus } & 137\end{array}$

7.3.5. Seleção de características 138

7.3.6. Mineração 141

8 Conclusões e Trabalhos Futuros 147

8.1. Conclusões 147

$\begin{array}{ll}\text { 8.2. Trabalhos Futuros } & 148\end{array}$

Referências Bibliográficas 150 


\section{Lista de Figuras}

$\begin{array}{ll}\text { Figura } 1 \text { - Processo de obtenção de conhecimento } & 17\end{array}$

Figura 2 - Integridade semântica de um SGBD 32

Figura 3 - Sites brasileiros quanto à frequência de modificação do conteúdo 35

Figura 4 - Coleções de documentos com elementos em comum 35

Figura 5 - Algumas estruturas sintáticas de um trecho de texto 36

Figura 6 - Documentos em formatos fracamente

estruturado e semiestruturado (respectivamente) 37

Figura 7 - Modelos de representação baseados em palavras e termos 40

Figura 8 - Multidisciplinaridade da Mineração de Textos 42

Figura 9 - Modelo simples de aprendizagem de máquina 45

Figura 10 - Linhas cronológica das etapas de um processo de

Mineração de Textos (por Aranha) 49

Figura 11 - Processo de representação estruturada de um texto 52

Figura 12 - Metodologia de identificação de tokens

proposta por KONCHADY 54

Figura 13 - Processo de tokenização seguido por remoção de stopwords 55

Figura 14 - Reconhecimento de anáfora com informações do contexto 57

Figura 15 - Erros de um processo de stemming:

overstemming e understemming $\quad 58$

Figura 16 - Derivações de um mesmo radical identificadas

pelo algoritmo de Porter $\quad 59$

Figura 17 - Representação de um índice invertido 64

Figura 18 - Estrutura básica de um Dicionário Thesaurus 65

Figura 20 - Gráfico de compensação entre precisão e abrangência 70

Figura 21 - Sistema Clássico de Recuperação de Informação 75

Figura 22 - Etapas possíveis no processo de Indexação

$\begin{array}{ll}\text { de documentos textuais } & 77\end{array}$

Figura 23 - Representação vetorial do documento Di

$\begin{array}{ll}\text { no espaço n-dimensional }(n=2) & 79\end{array}$

Figura 24 - Algoritmo KNN - Seleção baseada nos $k$ ( = 3) vizinhos 96 
Figura 25 - Máquina de Vetores de Suporte 98

Figura 26 - Abordagens SVM para problemas não binários 99

Figura 27 - Parâmetros de configuração do filtro

StringToWordVector do software Weka 104

Figura 28 - Interface de coleta do software STATISCA 108

Figura 29 - Classes gramaticais segundo a NGB 115

Figura 30 - Estrutura do dicionário Thesaurus utilizado no Sistema de MT 120

Figura 31 - Exemplo de documento do corpus CETENFolha 125

Figura 32 - Exemplo de modelagem de execução para o k-NN 127

Figura 33 - Exemplo de documento do corpus CETENFolha 131

Figura 34 - Exemplo de documento do corpus CETENFolha 131

Figura 35 - Representação de documentos na forma de bag of words 132

Figura 36 - Resultado do processo de remoção de stopwords

baseado em listas 133

Figura 37 - Resultado do processo de remoção de stopwords do domínio 134

Figura 38 - Identificação de classes gramaticais 135

Figura 40 - Fluxograma da lematização não verbal 137

Figura 41 - Substituição de termos por consulta ao Thesaurus 138

$\begin{array}{ll}\text { Figura } 42 \text { - Seleção de características } & 140\end{array}$

Figura 43 - Subconjuntos disponíveis 143

Figura 44 - Diagrama de estados 143

Figura 45 - Solução de melhor desempenho 145

Figura 46 - Desempenho lematização 146 


\section{Lista de Tabelas}

Tabela 1 - Resumo comparativo dos trabalhos relacionados quanto ao corpus, modelo de representação dos documentos e atribuição de pesos utilizados

Tabela 2 - Resumo comparativo dos trabalhos relacionados quanto a técnica de PLN, tarefa de MT e modelos de classificadores utilizados

Tabela 3 - As duas abordagens para a Análise de Textos e

suas principais Áreas de Conhecimento

Tabela 4 - As principais características de cada uma das

abordagens para a Análise de Textos

Tabela 5 - Marcação de tags para Reconhecimento de Entidades Nomeadas

Tabela 6 - Exemplo de classificações distintas de uma mesma entidade

Tabela 7 - Visualização das regras para concessão de empréstimos

em uma tabela

Tabela 8 - Comparação entre Recuperação de

Dados x Recuperação de Informação

Tabela 9 - Resultados conflitantes de SVMs binárias

Tabela 10 - Lista de cem stopwords utilizadas na

etapa de Pré-processamento

Tabela 11 - Exemplos ambíguos de identificação de classes gramaticais

Tabela 12 - Modelagem dos dados baseada em sliding window

Tabela 13 - Informações adicionais sobre o CETENFolha

Tabela 14 - Planejamento de ações

Tabela 15 - Planejamento de ações: Tokenização

Tabela 16 - Planejamento de ações: Remoção de stopwords

Tabela 17 - Planejamento de ações: PLN - Identificação de classes gramaticais

Tabela 18- Planejamento de ações: PLN - Lematização

Tabela 19- Planejamento de ações: PLN - Thesaurus

Tabela 20- Planejamento de ações: Seleção de características 
Tabela 22 - Configurações de execução do algoritmo SVM

Tabela 23 - Configurações de execução do algoritmo KNN

Tabela 24 - Configuração do melhor resultado obtido

Tabela 25 - Relação categoria x classificador 


\section{Lista de Equações}

Equação 1 - Teorema de Bayes 46

Equação 2 - Fórmula da métrica de desempenho "Precisão" 68

Equação 3 - Fórmula da métrica de desempenho "Abrangência" 68

Equação 4 - Fórmula da métrica de desempenho "Medida-F" 69

Equação 5 - Cálculo da medida TF em um documento 80

Equação 6 - Cálculo da medida TF-IDF em um documento 81

Equação 7 - Cálculo do escore de relevância de um termo 81

Equação 8 - Cálculo do Coeficiente de Correlação 82

Equação 9 - Cálculo do Ganho de Informação 84

Equação 10 - Cálculo de similaridade entre documentos por meio do 


\section{Lista de Siglas}

CT Categorização de Textos

ETL Extract Transform Load

IA Inteligência Artificial

KDD Knowledge Discovery in Databases

KDT Knowledge Discovery in Texts

$k$-NN k-Nearest Neighbors

NILC Núcleo Interinstitucional de Linguística Computacional

PLN Processamento de Linguagem Natural

RN Redes Neurais

SGBD Sistema Gerenciador de Bancos de Dados

SVM Support Vector Machine

VISL Visual Interactive Syntax Learning 